\title{
LABORATORY APPLIANCE: AN APPARATUS FOR MOTOR LEARNING RESEARCH
}

Luis Augusto Teixeira*

\section{ABSTRACT}

An apparatus developed for research in the motor learning area is presented in this paper. How it is built, its dimension and how it works is also explained. The device here presented is offered as an alternative to avoid

UNITERMS: Motor learning. Sport teaching.

The tasks utilized in motor learning studies have been found to be very close to one of the ends of a continuum. Tasks carried out in artificial conditions are at one pole and the ones carried out in natural conditions are at the other.

Tasks accomplished in artificial conditions refer to those especially developed for this experiment, such as the linear positioning of a slide or a lever. In order to isolate the variable that is being studied (independent) an experimental learning situation is usually built up. This situation is remote from the conditions in which the acquisition of motor skills usually takes place. An example of this is a situation in which the subject is blindfolded or a screen which prevents him from seeing the outcome of his movements is put before him.

One of the strongest reasons which leads researchers to adopt this kind of procedure is the demand for innovation of the task in motor learning studies; in other words, so there is no interference in the results of the test carried out, the task must be unknown to the subjects. the problem of polarization in situations of extreme artificiality or extreme naturality due to limitating factors, inherent to each of these methodological procedure lines.

Tasks carried out in natural learning conditions must be understood as being those naturally carried out by human beings, such as jumping, hurling, grasping, etc., learnt in a contest close to ordinary conditions of practice, namely, away from a laboratory (field situation).

The latter approach has been supported by scholars concerned in preserving the ecological effectiveness of the resulting knowledge. However, it is not exempted from criticism, inasmuch as through such procedures the control of variables is frequently quite impaired and considerably increases the number of intervenient variables, which make difficult the interpretation of results. Another criticism to this methodology refers to the demand for innovation of the task; once the researcher makes use of tasks carried out in the subjects' daily routine, he will not feel confident enough to report whether the results obtained were due to the procedure applied or to previous experiences.

Therefore deciding on the methodology to be carried out in a research, particularly in motor learning, is crucial. As far as 
methodology is concerned three aspects are particularly important: task, apparatus and procedure.

Having in mind the building up of motor skill experimental learning situations closer to an intermediate point between the extremes of artificial and natural situations, apparatus and task are suggested (used by TEIXEIRA, 1988), together with the most adequate procedures, so that researchers for the area may rely on a wider spread of choices when selecting or working out the methodology.

We shall now procede with a description of how the apparatus is built, its dimension and how it works.

The apparatus is made of wood and it has two moving parts, the support and the main part. Its shape is that of a $73 \mathrm{~cm}$ high $x$ $1.20 \mathrm{~cm}$ long $\times 1.20 \mathrm{~cm}$ wide table, its surface limited on both sides and at the back part by $10 \mathrm{~cm}$ high vertical borders. Surface and borders are $2 \mathrm{~cm}$ thick (see Figure 1).

On the surface there are four impelling zones, with a $3 \mathrm{~cm}$ diameter, sequently arranged over the median longitudinal axle, $10 \mathrm{~cm}$ apart from one another as from the limit of the front part to the surface of the apparatus. The first impelling zone is $15 \mathrm{~cm}$ distant from this limit.

From the geometrical centre of the third target, an arc having a $40 \mathrm{~cm}$ radius is traced, over which five circular targets with a $5 \mathrm{~cm}$ diameter are drawn, $20 \mathrm{~cm}$ distant from

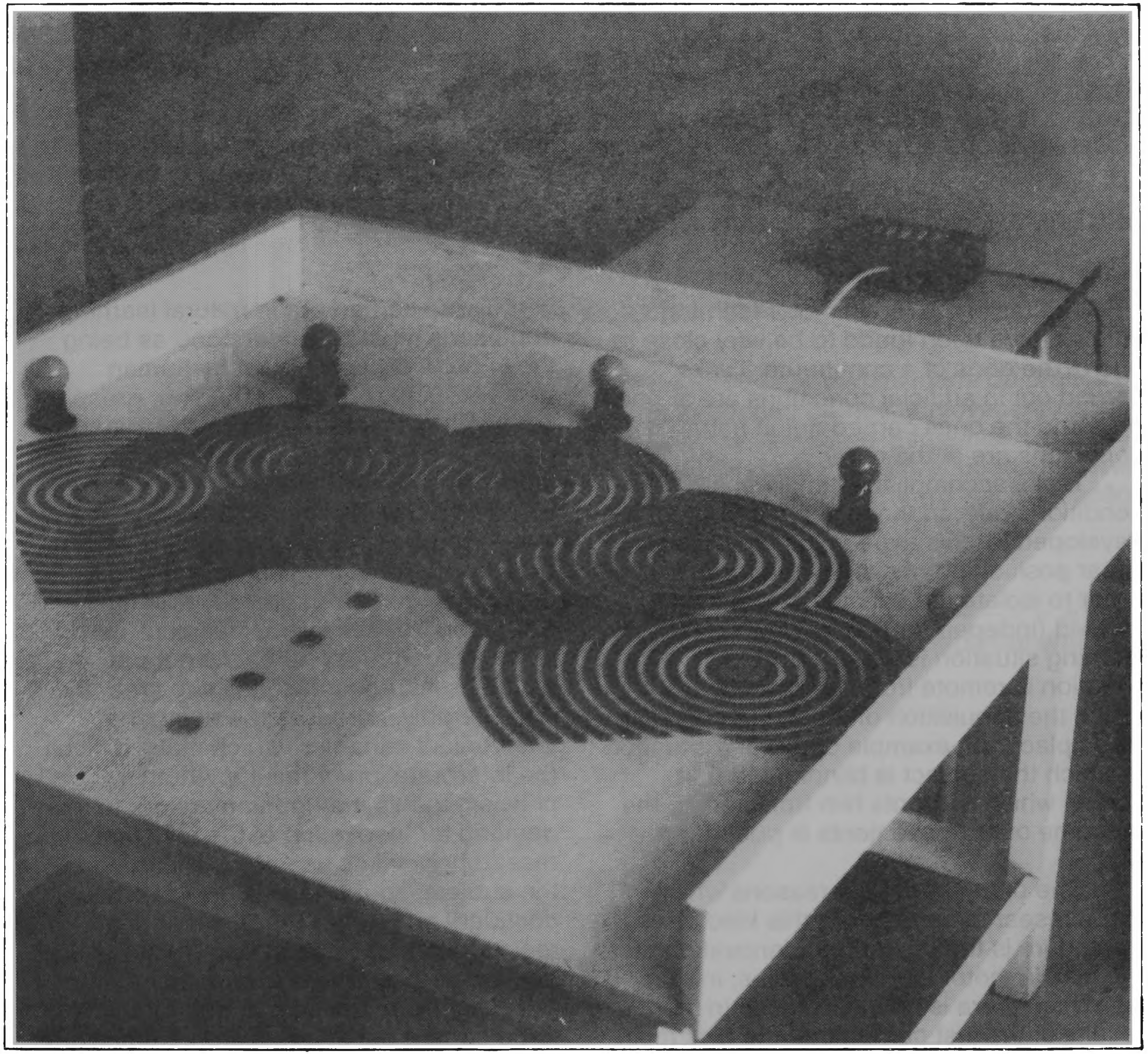

Figure 1 - Perspective view of the apparatus 
one another. There is a central target, another two on the right and two on the left. Each target is surrounded by ten complete concentric circular areas and another twenty five incomplete concentric circular areas (fifteen towards the impelling zones and ten towards the back part of the surface of the apparatus). The complete and incomplete circular areas are $1 \mathrm{~cm}$ wide and arranged in a sequential way.

Behind every set of back incomplete circular area, on the surface of the apparatus, there is a 15 watt bulb alternately yellow and orange in colour. Each bulb is connected to the switch board, placed at the back of the apparatus, beyond the limits of its surface. The switch board consists of five switches arranged in a small metal box - each switch lighs up a given bulb. (see Figures 1 and 2).

All above mentioned features of the apparatus are obtained through the following building process:

(a) both components of the set are painted with oil paint;

(b) the main part (upper) is drawn and painted with black atomic brushes (error measurement targets and areas) and red (impelling zones);

(c) the error measurement areas are numbered with $0.3 \mathrm{~cm}$ black sticking digits;

(d) a perforation is made behind every error delimitation zone, corresponding to each target; (e) the switch board is coupled;

(f) the eletric part consisting of copper wires, supports for the 15 watt bulbs is installed; one bulb is installed on each perforation behind every target;

(g) the surface of the main body is covered with colorless varnish; and

(h) the surface is the covered with liquid wax.

Impelling instruments are built to complement the apparatus. They are made of transparent colorless plastic, small lead spheres (to increase weight) and durepox plus (to make the support equipment). These impelling instruments are $1.2 \mathrm{~cm}$ high, and have a $3 \mathrm{~cm}$ diameter and a 7.7 gram mass. On the upper surface there is a circular support, and on the lower surface there is a spot showing its geometrical centre. (see Figure 2)

This apparatus requires the subject to be seated facing the front area (the impelling zone area), with the shoulder of the predominant arm in line with the impelling zones and the instrument placed in one of these zones. This done, the experimenter starts the switch board, lighting up one of the bulbs, which indicates the target to be struck.

The subject's task is to impel the instrument by stretching the index finger of the predominant hand, which rests on the

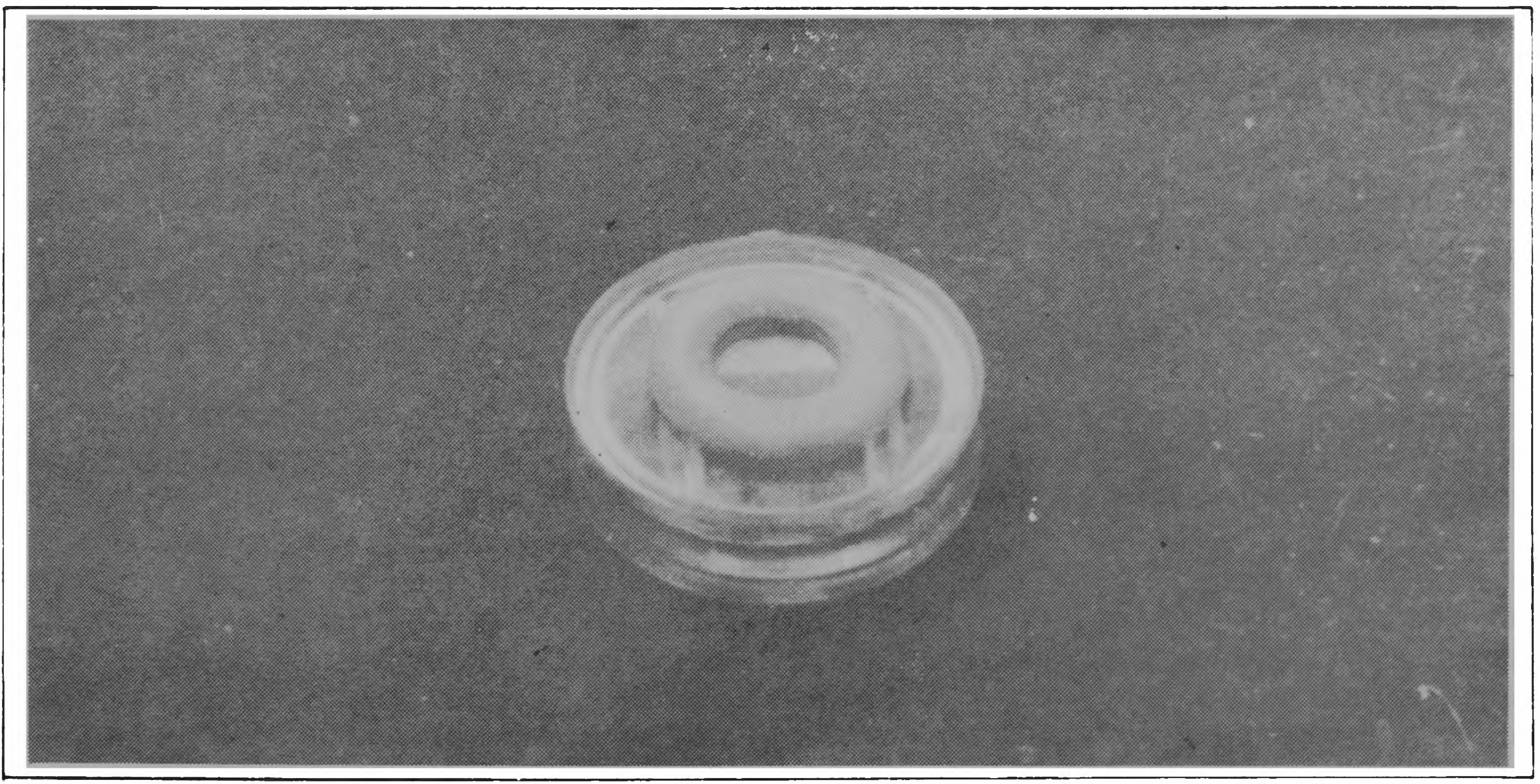

Figure 2 - Impelling instrument 
upper area of the instrument (circular support), trying to make it stop on the indicated target.

The experimenter evaluates the result, with the help of error measurement areas, and a few seconds prior to the ending of the inter attempt intervals he replaces the instrument on the adequate impelling zone, and gives continuity to the data colletcting process. Such procedures are repeated attempt after attempt, until the desired amount of practice is reached.

\section{CONCLUSION}

Through the use of such an apparatus and procedures, the learning experimental conditions presented are considered to be distant from the extremes of "artificiality" and "naturality" and are placed at a spot closer to the intermediate point. They provide learning in a laboratory where the subject collects the entering information, processes and integrates it to previous experiences, works out his action plan with due specifications, carries it out, observes the results and evaluates his answer. Such situation when analysed under these aspects, reproduces the ordinary learning process, not ignoring the control of important variables which may interfere with the results of the study.

These features of the apparatus are considered appropriate for research lines focused on motor learning theories (ADAMS, 1971; SCHMIDT, 1975), particularly in relation to the specificality hipothesis test (ADAMS) versus variability (SCHMIDT) of practice, and on the contextual interference hypothesis test (Batting, quoted in SHEA \& MORGAN, 1979), which bring forward fundamental points for the advance of current motor learning knowledge..

* Assistant Teacher at The School of Physical Education, Department of Gymnastics, University of São Paulo

The references are found in the article in Portuguese. 